\title{
Automatic aortic root segmentation and anatomical landmarks detection for TAVI procedure planning
}

Florent Lalys ${ }^{1}$, Simon Esneault ${ }^{1}$, Miguel Castro ${ }^{2,3}$, Lucas Royer ${ }^{1}$, Pascal Haigron ${ }^{2,3}$, Vincent Auffret ${ }^{2,3,4}$, Jacques Tomasi ${ }^{4}$

1. Therenva, F-35000, Rennes, France

2. INSERM, U1099, F-35000 Rennes, France

3. University Rennes 1 , Signal and Image Processing Laboratory (LTSI), Rennes, France

4. CHU Rennes, Department of Cardiothoracic and Vascular Surgery, Rennes, France

\begin{abstract}
Purpose. Minimal invasive trans-catheter aortic valve implantation (TAVI) has emerged as a treatment of choice for high-risk patients with severe aortic stenosis. However, the planning of TAVI procedures would greatly benefit from automation to speed up, secure and guide the deployment of the prosthetic valve. We propose a hybrid approach allowing the computation of relevant anatomical measurements along with an enhanced visualization.
\end{abstract}

Methods. After an initial step of centerline detection and aorta segmentation, model-based and statistical-based methods are used in combination with 3D active contour models to exploit the complementary aspects of these methods and automatically detect aortic leaflets and coronary ostia locations. Important anatomical measurements are then derived from these landmarks.

Results. A validation on 50 patients showed good precision with respect to expert sizing for the ascending aorta diameter calculation $(2.2+/-2.1 \mathrm{~mm})$, the annulus diameter $(1.31+/-0.75 \mathrm{~mm})$, and both the right and left coronary ostia detection $(1.96+/-0.87 \mathrm{~mm}$ and $1.80+/-0.74 \mathrm{~mm}$, respectively). The visualization is enhanced thanks to the aorta and aortic root segmentation, the latter showing good agreement with manual expert delineation (Jaccard index: $0.96+/-0.03$ ).

Conclusions. This pipeline is promising and could greatly facilitate TAVI planning.

Keywords: TAVI; statistical-based methods; active contour models; endovascular procedures 


\section{Introduction}

Minimal invasive trans-catheter aortic valve implantation (TAVI) is an emerging technique that is especially suitable for high-risk patients with severe aortic stenosis, but that has also the potential to be applied to lower surgical-risk patients [1]. However, exact targeting remains very important, since complications can appear due to a misplaced valve. Adverse effects arising from misplaced valve can be reduced with improved patient selection and aortic sizing. Specifically, it is crucial to decide the suitable type and dimensions of prosthesis based on the aortic root geometry. For this type of pre-procedural planning, computed tomography angiography (CTA) scan has been accepted as the modality of choice.

Using CTA, a number of studies have proposed imaging pipelines to extract the aortic valve anatomical parameters and derived clinical measurements. While solutions using manual interactions have been first proposed to guide TAVI planning (e.g. [2,3]), automatic planning can reduce the inconsistency in sizing due to inter-observer variability [4]. From the TAVI planning, many steps can be independently decomposed, from the segmentation of the thoracic aorta to the automatic detection of key anatomical landmarks. For the thoracic aorta segmentation, multi-atlas-based segmentation with Hough transform have been proposed in noncontrast CT [5] or with 3D level set approaches [6] in CTA. For aortic root segmentation, 3D normalized cuts [7,8] have been used. Korosoglou et al. [9] used a model-based segmentation approach to highlight the usefulness of software-assisted pre-procedural assessment, while Gao et al. [10] automatically segment the aortic root following an atlas-based approach.

Automatic detection of landmarks is essential for TAVI planning but often laborious to extract. From these measurements, annulus radius and orientation, and distance from annulus plane to right and left coronary ostia can be derived. Zheng et al. [11,12] proposed a learningbased approach to automatically detect major landmarks with marginal space learning based on the C-arm CT. Model-based approach has been followed by Waechter et al. [13] to locate 
coronary ostia and annulus plane. Ionasec et al. [14] also proposed a valve model to detect landmarks but their work was based on 4D CT. Automatic measurements of aortic annulus diameters has been proposed with 3d transesophageal echocardiography (TEE) [15] or ultrasound images [16-18]. Grbic et al. [19] employed robust machine learning algorithms to estimate the valve model parameters from non-contrast CT including information on valve leaflets and calcium. Segmentation of aortic valve from TEE using an improved probability estimation and continuous max-flow approach has also been proposed [20], or using a combination of shape-based B-Spline explicit active surface and generalized Hough transform [21].

From the literature it has been demonstrated that automated landmarks detection allows standardizing the planning, reducing inter-observer variation and reducing sizing time. While specific aspects of the TAVI planning have been mostly independently studied so far, and

often with imaging modalities that are not adopted by the cardio-vascular community, there is no standardized automated solution that encompass the different steps that compose the TAVI planning, i.e. the segmentation of the thoracic aorta, the extraction of aorta centerlines, the aortic root segmentation and the major anatomical landmarks detection. The purpose of this work is to propose a complete TAVI planning tool based on an image processing pipeline that exploits the complementary aspects of state-of-the-art detection approaches.

\section{Material and methods}

The proposed image-processing pipeline consists of 4 main steps and is depicted in Fig. 1. The input data is the pre-procedural electrocardiographic (ECG)-gated CTA, widely adopted by cardio-vascular surgeons. It is loaded into the EndoSize ${ }^{\circledR}$ software [22], a CE- and FDAmarked validated medical device for planning and sizing of endovascular procedures. After the initial centerline extraction and aorta segmentation, a method for automatic detection of aortic leaflets is presented, as well as a method for the automatic detection of coronary artery 
ostia. For enhanced 3D visualization and landmarks adjustment, we also propose a 3D segmentation of the aortic root. The final step includes a refinement of anatomical landmarks position. Each step is independently presented and validated using a large dataset of patients operated for TAVI.

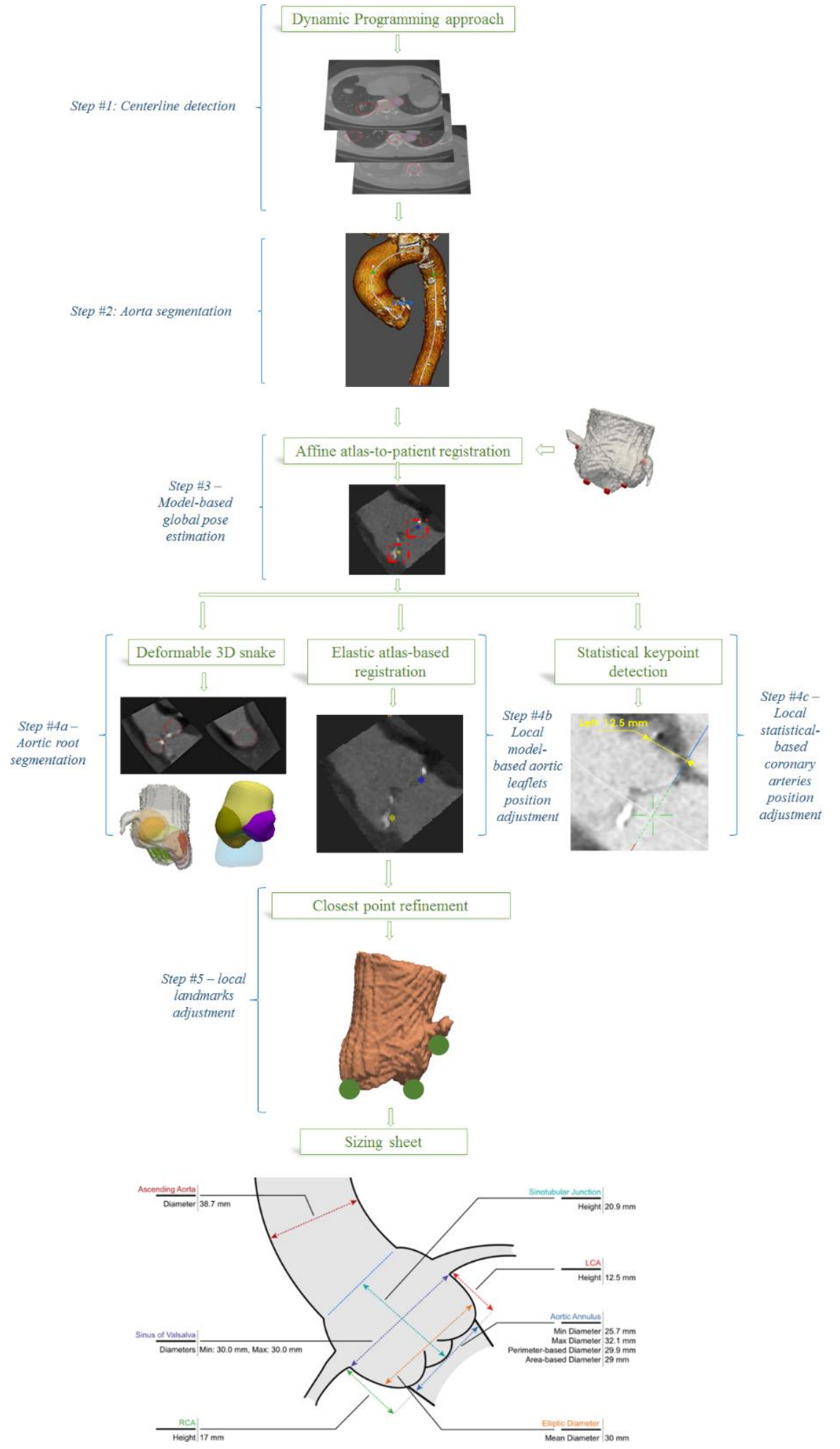

Fig. 1: Workflow of segmentation/detection within the TAVI planning software 


\subsection{Step \#1 \& step \#2 - Centerline extraction \& aorta segmentation}

The only required user interaction is the placement of one seed point approximatively set in the aortic root. A volume of interest (VOI) is set in order to keep the ascending aorta, the aortic arch and the descending aorta, using volumes resolution of $1 \mathrm{x} 1 \mathrm{x} 1 \mathrm{~mm}$. Then, after a rough segmentation of the whole aorta using binary threshold (100 HU to $600 \mathrm{HU}$ ), a dynamic programming approach is applied in the Hough space for localizing key center points in the aorta. We exploit here the 3-D tubular shape characteristic of the aorta, where appearance of the ascending and descending aortas in axial slices approximates a circular shape. The objective is to choose one point in the Hough accumulator of each axial image. A dynamic programming approach is employed to find the optimal combination of points from the Hough accumulators by taking both the center coordinates and the radius as input parameters. We finally used a fast-marching-based minimal path extraction to refine the centerline detection in case of errors.

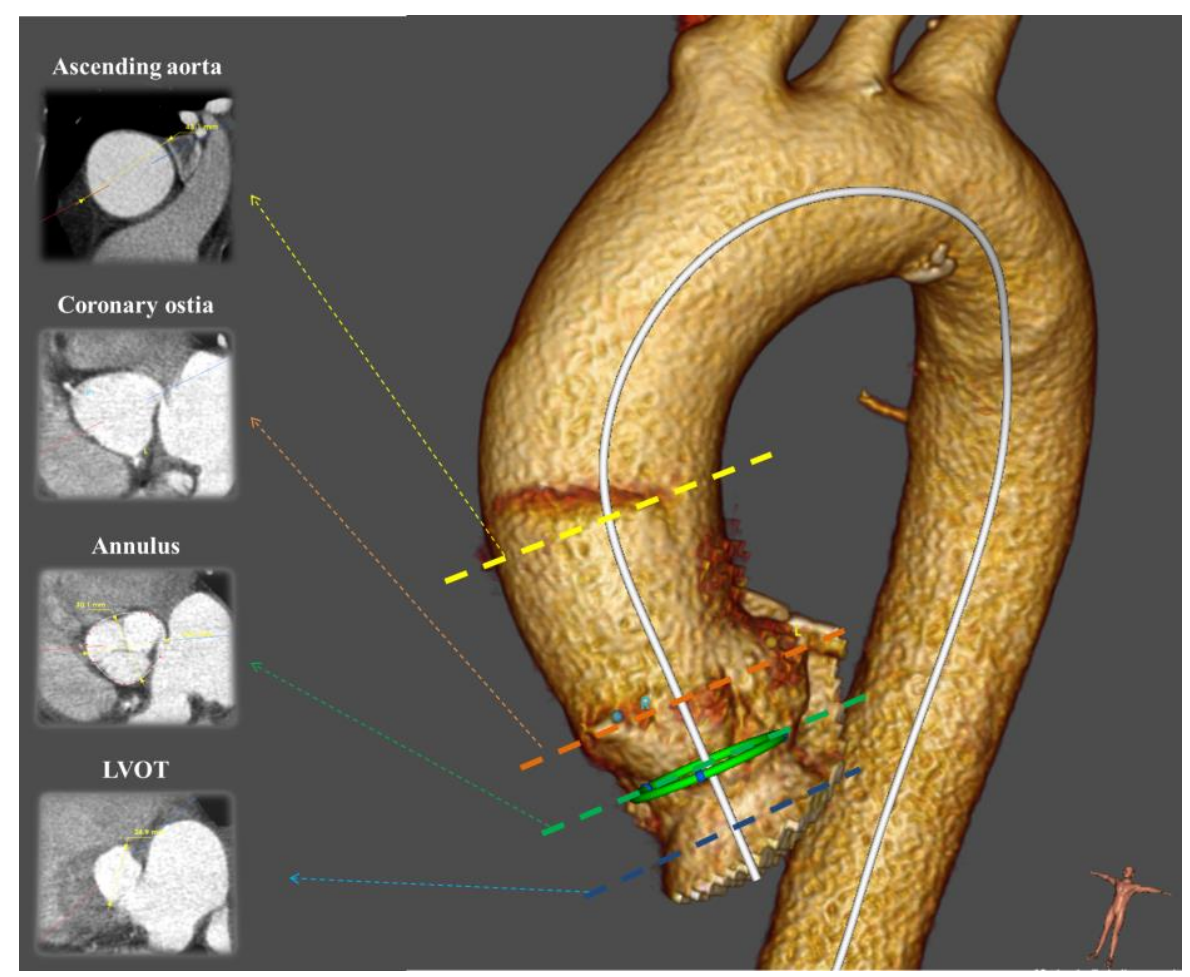

Fig. 2: Visualization of the different aorta levels 
Starting from the detected centerline, the aorta is also automatically segmented. As this step has already been presented in a previous paper [22], it will not be developed in detail here. This segmentation of the upper part of the aorta is mainly used for visualization purposes (Fig. 2.), but is also useful to determine the ascending aorta diameter, which is a key parameter for TAVI planning. Min, max and mean diameter of each slice perpendicular to the centerline are made available to the user.

\subsection{Step \#3 - Global pose estimation}

The processing pipeline then focuses on the aortic root. From the final segmented aorta, its centerline, and the user seed point, a second VOI centered on the aortic root is derived with a tube mask of constant diameter (computed according to the maximum aorta diameter) along the centerline and smoothed using anisotropic diffusion. Then, the global pose of the aortic root was estimated following a model-based approach. Due to computation time constrain, a single low resolution atlas (average model) of the aortic root was constructed offline using 25 volumes following the standard iterative methodology of atlas creation [23], resulting in a volume with both an average unbiased shape and average intensity (size 26x 26x 26, 2mm isotropic resolution). Internal experiments were conducted and showed that from 25 patients, the accuracy of the global pose didn't significantly change. Eight keypoints were positioned on patient's images by an expert and warped into the atlas coordinate system: the left and right coronary ostia, the three aortic cusps leaflets and three seed points approximatively corresponding to the barycenter of the cusps. Then, the keypoints information are transferred to the patient coordinate system by affine intensity-based registration algorithm (metric: crosscorrelation, optimizer: Powell, isotropic scaling), where calcifications were masked out using a simple threshold (intensity $>800 \mathrm{HU}$ ). From the position, orientation, and scale of the aver- 
age shape, we can infer a first rough estimation of the position of each of the 8 anatomical keypoints.

\subsection{Step \#4 - Local landmarks detection \& aortic root segmentation}

The 8 keypoints are further either refined or directly used as seed points under the guidance of their own specific detectors.

- Step \#4a - aortic root segmentation: Starting from $2 \mathrm{~mm}$ diameter spheres initialized from seed points in the barycenter of the three cusps (computed from step \#3), 3D deformable models were employed to accurately segment the aortic root by constraining smooth boundaries. Specifically, a 3D gradient vector flow snake [24] with a descent gradient optimizer was employed, with proper internal and external energy tuned to keep a smooth curvature along the cusps (alpha $=0.1$, beta $=3$, gamma $=0.01$, number of iterations $=200$ ). Combining these segmentations allows extracting the entire aortic root, and is useful to remove all unnecessary arterial structures (e.g. heart, coronary arteries) for proper visualization.

- Step \#4b - leaflet position adjustment: Similar to hierarchical approaches, and using the positions initialized in step \#3, the three aortic cusps leaflets are refined in a smaller $2 \mathrm{~cm}$ diameter VOI. Three high resolution atlases (size $26 \times 26 \times 26,0.6 \mathrm{~mm}^{3}$ isotropic resolution) were constructed using the same learning database of 25 patients, and deformable registration (diffeomorphic demons' algorithm, cross-correlation similarity metric, gradient descent optimizer, rigid registration initialization) was used to warp final landmark positions on the different patient local VOIs.

- Step \#4c - coronary ostia position adjustment: A training phase is necessary to learn the relationship between the image and the coronary ostia location. We used a combination of 3D Haar-like features, histogram of gradient (HoG) and speeded up robust features (SURF) 
trained using a Support Vector Machine (gaussian kernel) on an external database of 100 patients originally intended for thoracic endovascular aneurysm repair (TEVAR). Even if patients treated with TAVI have usually more calcifications than patients with TEVAR, no significant differences exist for coronary anatomy between both groups. The correct position is automatically detected on the new volume by restraining the area of research in a smaller $2 \mathrm{~cm}$ diameter VOI around the positions estimated from step \#3.

\subsection{Step \#5 - Landmarks position refinement}

To take advantage of all previous methods, results from the different steps were combined. Landmark positions found in step \#4b and step \#4c were further locally refined using the aortic root segmentation from step \#4a, which is well suitable to precisely detect boundaries. Specifically, landmarks were adjusted to the closest point of the 3D aortic root surface. Important TAVI measurements were then derived from these landmarks. The annulus plane was defined as the plane connecting the three leaflets. Given the annulus plane, the annulus to ostia distance was also computed for both coronary ostia.

\subsection{Evaluation}

Anonymized electrocardiogram-gated CTA scans (Siemens Healthcare, Munich, Germany) with resolution of $0.87 \times 0.87 \times 0.62 \mathrm{~mm}, 75 \mathrm{~mL}$ of contrast media, from 50 symptomatic patients $(80+/-5$ years old, $65 \%$ male $)$ originally intended for pre-operative planning of TAVI at the Rennes University Hospital were systematically obtained during a 1 year period between 2015 and 2016. All patients had a tricuspid valve. All steps were independently evaluated. Centerline detection was qualitatively assessed. To be correctly detected, a centerline has to include the ascending aorta, the aortic arch and the descending aorta and has to have all points within the aorta. For the aorta segmentation, we computed the maximum ascending 
aorta diameter, which is another important measure to derive from the planning. The aortic root landmarks detection was compared to a manual expert delineation throughout the pipeline to evaluate the influence of each step. Due to the high inter-observer variability, the same expert (an engineer but with a long background in TAVI planning) annotated the training and test patients. In addition to the position of the landmarks, the annulus radius and the annulus to ostia heights were also evaluated. Impact of each step was iteratively validated using a paired sample t-test. Finally, the accuracy of the aortic root segmentation was assessed by comparing the segmentation results with manual delineations using the Jaccard index.

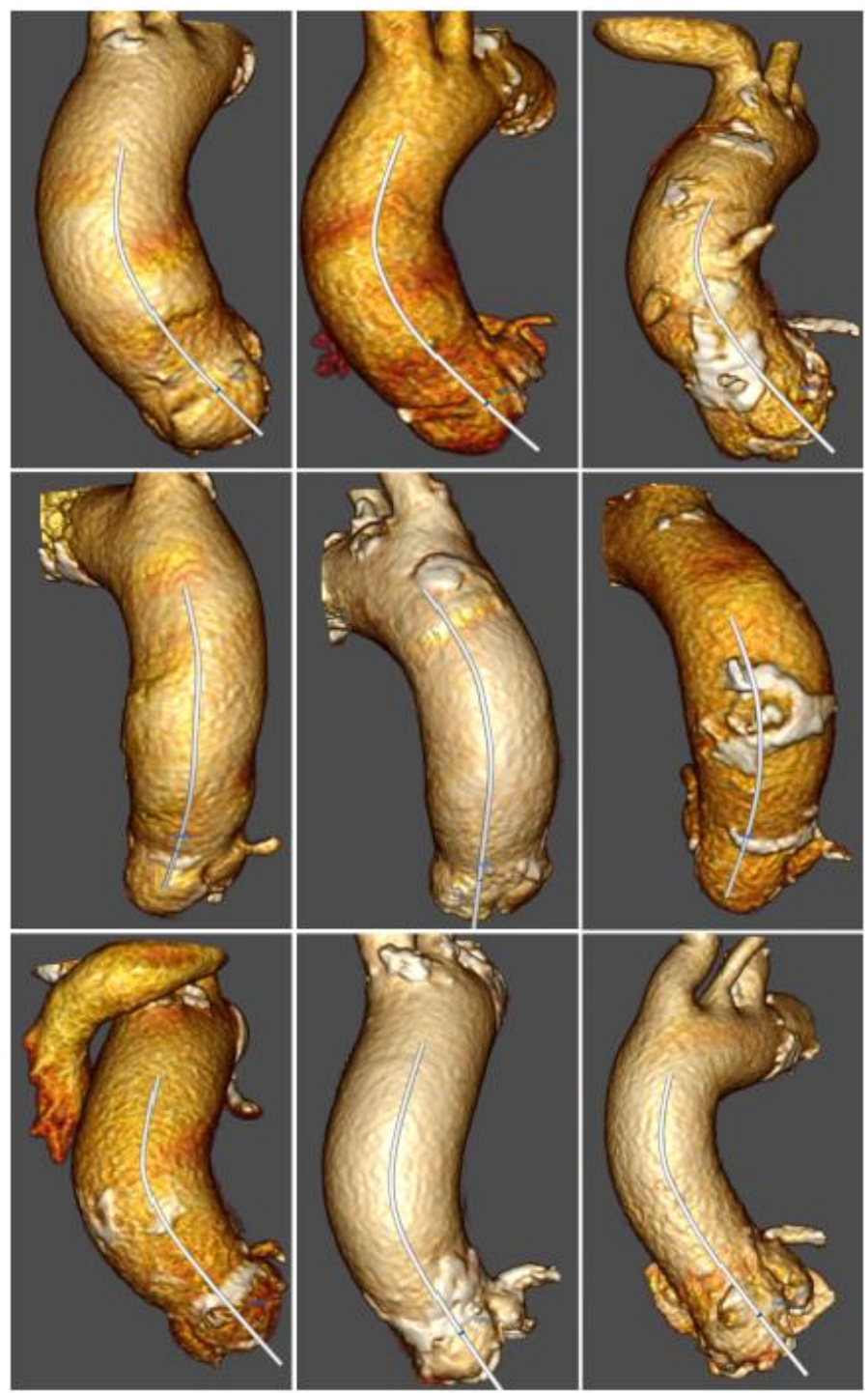

Fig. 3: Examples of centerlines extraction and aorta segmentation for 9 patients 


\section{Results}

\subsection{Centerline detection \& aorta segmentation}

Aorta centerlines from 48 patients out of 50 were correctly extracted based on the initial seed point only (Fig. 3.). For the two remaining patients, very late contrast injections were noticed that highlight other neighboring structures. Qualitatively, aorta segmentations were also conclusive for the entire database (Fig. 3.), and an error of $2.2+/-2.1 \mathrm{~mm}$ was found when comparing the automatic maximum ascending aorta diameter with the expert sizing.

\subsection{Anatomical landmarks detection}

Landmarks detection showed very accurate results for the aortic leaflets detection, and accurate results for the coronary ostia detection (Fig. 4.). Each additional step showed statistically lower errors than the previous step $(\mathrm{p}<0.05)$. Aortic leaflets detection showed error of $1.96+/-$ $0.84 \mathrm{~mm}, 2.34+/-0.78 \mathrm{~mm}$ and $1.23+/-1.12 \mathrm{~mm}$ for the right, left and non-coronary ostia, respectively. The annulus diameter, derived from the aortic leaflet detection and the centerline, showed an error of $1.31+/-0.75 \mathrm{~mm}$, while right and left coronary ostia heights showed errors of $1.96+/-0.87 \mathrm{~mm}$ and $1.80+/-0.74 \mathrm{~mm}$, respectively.

\subsection{Aortic root segmentation}

The aortic root segmentation showed accurate results compared to manual delineations: average Jaccard index of $0.96+/-0.03$, average false positive ratio of $4.0+/-2.3 \%$ and an average false negative ratio of $0.6+/-0.2 \%$. 


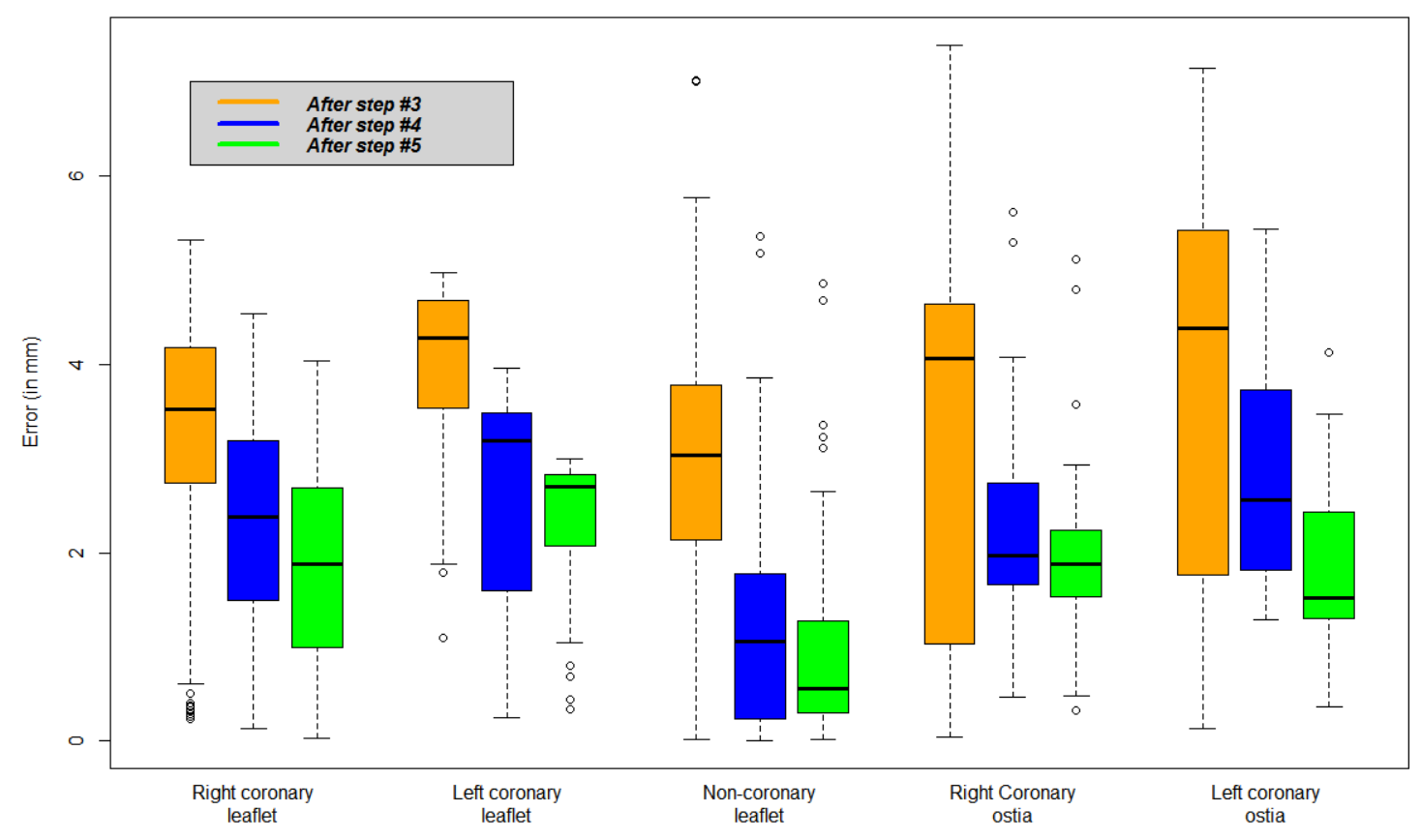

Fig. 4: Difference (in $\mathrm{mm}$ ) between automatic landmark detection and expert positioning at each step of the pipeline

\section{Discussion}

A TAVI planning tool with automatic measurements is presented that allows for simplified planning. One simple user interaction is needed to directly access the dedicated VOI and retrieve a list of key anatomical measurements, which considerably reduces user interactions compared to traditional TAVI sizing. In certain cases, and according to physician's practice, slight adjustments might be necessary. The visualization is also simplified thanks to the aortic root segmentation, allowing the generation of additional visualization modalities such as valve close-up, half pipe or calcification view (Fig. 5.). The pipeline showed descent computation burden, with an average total processing time of less than 8 s on a $3.0 \mathrm{GHz}$ Quad Core 2 CPU, totally compatible with clinical routine. The next step of the software includes the choice of access sites before the generation of a summarized sizing sheet. A set of commercial prosthetic aortic valve is then proposed to be able to choose the most suitable one. This step is 
of high importance because of the recent development of a large set of available valve prosthesis (e.g. [25]). A clinical study is currently under consideration to evaluate the time and accuracy obtained with/without the use of this automatic pipeline.

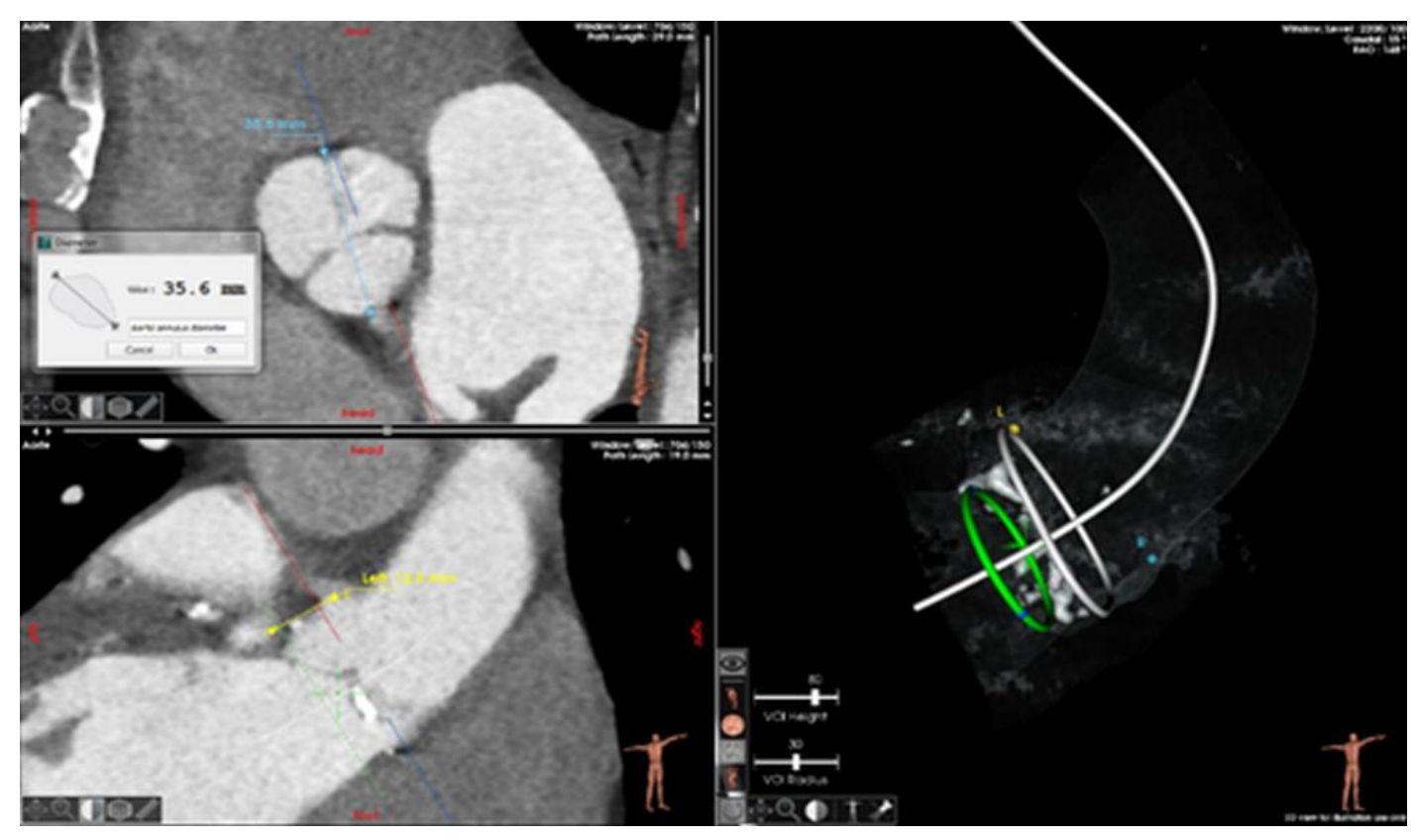

Fig. 5: Visualization of the measurement step within the EndoSize ${ }^{\circledR}$ TAVI module

The choice of using various approaches for detecting anatomical landmarks was driven by two main observations: first, model-based approaches may not be adapted for VOIs including arteries, as the registration is driven by the aortic root boundaries more than by arteries. Second, even if supervised discriminative machine learning techniques are robust to different field-of-views and contrast injections, it is not as accurate when dealing with strong intensity differences (i.e. calcifications). Due to the high anatomical variability, our database of 100 patients was not sufficient for this problem. For our aortic root segmentation method, active contour models are robust to noise and less sensitive to large intensity gaps than other image processing methods. Step \#5 was therefore useful to constrain landmarks on the aortic root surface. The succession of steps was validated by systematically quantifying errors for each landmark and the derived measures. 
Compared to other studies, our current framework demonstrated similar accuracies, but proposes a wide set of automatic measurement and visualization tools that no study has presented so far. While segmentations are difficult to compare due to database heterogeneity or metrics used, it is however possible to confront our landmark detection results with the literature. For aortic leaflets detection based on C-arm CT, Zheng et al. [11,12] achieved a mean detection error of $2.09+/-1.18 \mathrm{~mm}$, and a $2.07+/-1.53 \mathrm{~mm}$ detection error for coronary ostia, which is very close from our results. Using pre-operative CT, Waechter et al. [13] obtained impressive accuracy for coronary ostia detection (error of $1.2+/-0.6 \mathrm{~mm}$ for the left ostia, $1.0+/-0.8 \mathrm{~mm}$ for the right ostia), however the validation is performed only on 20 patients and no results on centerline detection and ascending aorta segmentation is proposed. Elattar et al. [26] reported a $2.81+/-2.08 \mathrm{~mm}$ error between automatic landmarks detection and the reference landmarks, comparable with the inter-observer variability of $2.38+/-1.56 \mathrm{~mm}$, while Liang et al. [27] reported a mean error of $0.69 \mathrm{~mm}$ for aortic valve leaflets detection. Considering the high inter-observer variability discussed in the work of Zhao et al. [4] and Elattar et al. [26], a TAVI sizing can be considered accurate enough with results below $3 \mathrm{~mm}$, which is the case in this study.

This study suffers from some limitations. First, the manual delineation has been performed by a single surgeon, while intra- and inter-expert variability would have been informative. The on-going clinical study includes three surgeons of different experience level and will allow us to quantify those variabilities. Second, this workflow is well suitable for patients with a tricuspid valve, and the lack of patients with bicuspid valves prevented us to train and validate a specific workflow. Finally, the set of parameters used for the different processing steps has been tuned and validated on clinical data from one single site, and could be not fully optimized when experimented on CTA with different contrast injection protocols or resolution. 


\section{Conclusion}

We proposed and evaluated a hybrid approach for TAVI planning allowing the computation of relevant anatomical measurements. After a minimal user interaction, it is possible to detect the aorta centerline, segment the aorta, detect key anatomical landmarks and segment the aortic root in an automatic fashion. The first model-based pose estimation, the atlas-based leaflets detection, the statistical-based coronary ostia detection, and the automatic segmentation of the aortic root are suitable to directly access the dedicated VOI, propose a set of automatic measurements and enhance the visualization of the aortic root. With an upcoming clinical evaluation, the proposed pipeline is promising and could simplify TAVI planning.

Conflict of interest: This research is sponsored by Therenva and may lead to the development of products. I have disclosed those interests fully to Taylor \& Francis, and have in place an approved plan for managing any potential conflicts arising from this arrangement. 


\section{References}

1 Lindman BR, Pibarot P, Arnold SV, Suri RM, McAndrew TC, Maniar HS, et al.: Transcatheter versus surgical aortic valve replacement in patients with diabetes and severe aortic stenosis at high risk for surgery: an analysis of the PARTNER Trial (Placement of Aortic Transcatheter Valve). J Am Coll Cardiol 2014;63:1090-1099.

2 Gessat M, Merk DR, Falk V, Walther T, Jacobs S, Nöttling A, et al.: A planning system for transapical aortic valve implantation; in Miga MI, Wong KH (eds). 2009, p 72611E.

3 Karar ME, Gessat M, Walther T, Falk V, Burgert O: Towards a new image guidance system for assisting transapical minimally invasive aortic valve implantation. Conf Proc Annu Int Conf IEEE Eng Med Biol Soc IEEE Eng Med Biol Soc Annu Conf 2009;2009:3645-3648.

4 Zhao F, Xie X, Roach M: Computer Vision Techniques for Transcatheter Intervention. IEEE J Transl Eng Health Med 2015;3:1900331.

5 Isgum I, Staring M, Rutten A, Prokop M, Viergever MA, van Ginneken B: Multi-AtlasBased Segmentation With Local Decision Fusion \#x2014;Application to Cardiac and Aortic Segmentation in CT Scans. IEEE Trans Med Imaging 2009;28:1000-1010.

6 Kurugol S, San Jose Estepar R, Ross J, Washko GR: Aorta segmentation with a 3D level set approach and quantification of aortic calcifications in non-contrast chest CT. Conf Proc Annu Int Conf IEEE Eng Med Biol Soc IEEE Eng Med Biol Soc Annu Conf 2012;2012:2343-2346.

7 Elattar MA, Wiegerinck EM, Planken RN, Vanbavel E, Assen HC van, Jr JB, et al.: Automatic segmentation of the aortic root in CT angiography of candidate patients for transcatheter aortic valve implantation. Med Biol Eng Comput 2014;52:611-618.

8 Elattar M, Wiegerinck E, Planken N, Vanbavel E, Assen H van, Jr JB, et al.: Automated Normalized Cut Segmentation of Aortic Root in CT Angiography; in Romero LMR (ed): XIII Mediterranean Conference on Medical and Biological Engineering and Computing 2013. Springer International Publishing, 2014, pp 1821-1824.

9 Korosoglou G, Gitsioudis G, Waechter-Stehle I, Weese J, Krumsdorf U, Chorianopoulos E, et al.: Objective quantification of aortic valvular structures by cardiac computed tomography angiography in patients considered for transcatheter aortic valve implantation. Catheter Cardiovasc Interv Off J Soc Card Angiogr Interv 2013;81:148-159.

10 Gao X, Kitslaar PH, Scholte AJHA, Lelieveldt BPF, Dijkstra J, Reiber JHC: Automatic aortic root segmentation in CTA whole-body dataset2016, pp 97850F-97850F-7.

11 Zheng Y, John M, Liao R, Nottling A, Boese J, Kempfert J, et al.: Automatic Aorta Segmentation and Valve Landmark Detection in C-Arm CT for Transcatheter Aortic Valve Implantation. IEEE Trans Med Imaging 2012;31:2307-2321.

12 Zheng Y, John M, Liao R, Boese J, Kirschstein U, Georgescu B, et al.: Automatic aorta segmentation and valve landmark detection in C-arm CT: application to aortic valve implantation. Med Image Comput Comput-Assist Interv MICCAI Int Conf Med Image Comput Comput-Assist Interv 2010;13:476-483. 
13 Waechter I, Kneser R, Korosoglou G, Peters J, Bakker NH, van der Boomen R, et al.: Patient specific models for planning and guidance of minimally invasive aortic valve implantation. Med Image Comput Comput-Assist Interv MICCAI Int Conf Med Image Comput Comput-Assist Interv 2010;13:526-533.

14 Ionasec RI, Georgescu B, Gassner E, Vogt S, Kutter O, Scheuering M, et al.: Dynamic model-driven quantitative and visual evaluation of the aortic valve from 4D CT. Med Image Comput Comput-Assist Interv MICCAI Int Conf Med Image Comput Comput-Assist Interv 2008;11:686-694.

15 Bersvendsen J, Beitnes JO, Urheim S, Aakhus S, Samset E: Automatic measurement of aortic annulus diameter in 3-dimensional transoesophageal echocardiography. BMC Med Imaging 2014;14:31-35.

16 Dong B, Guo Y, Wang B, Gu L: Aortic valve segmentation from ultrasound images based on shape constraint CV model. Conf Proc Annu Int Conf IEEE Eng Med Biol Soc IEEE Eng Med Biol Soc Annu Conf 2013;2013:1402-1405.

17 Guo Y, Dong B, Wang B, Xie H, Zhang S, Gu L: Semiautomatic segmentation of aortic valve from sequenced ultrasound image using a novel shape-constraint GCV model. Med Phys 2014;41:072901.

18 Nie Y, Luo Z, Cai J, Gu L: A novel aortic valve segmentation from ultrasound image using continuous max-flow approach. Conf Proc Annu Int Conf IEEE Eng Med Biol Soc IEEE Eng Med Biol Soc Annu Conf 2013;2013:3311-3314.

19 Grbic S, Ionasec R, Mansi T, Georgescu B, Vega-Higuera F, Navab N, et al.: Advanced intervention planning for Transcatheter Aortic Valve Implantations (TAVI) from CT using volumetric models; in : 2013 IEEE 10th International Symposium on Biomedical Imaging. 2013, pp 1424-1427.

20 Cai J, Zhuang X, Nie Y, Luo Z, Gu L: Real-time aortic valve segmentation from transesophageal echocardiography sequence. Int $\mathrm{J}$ Comput Assist Radiol Surg 2015;10:447-458.

21 Queiros S, Papachristidis A, Morais P, Theodoropoulos KC, Fonseca JC, Monaghan MJ, et al.: Fully automatic 3D-TEE segmentation for the planning of Transcatheter Aortic Valve Implantation. IEEE Trans Biomed Eng 2016;PP:1-1.

22 Kaladji A, Lucas A, Kervio G, Haigron P, Cardon A: Sizing for endovascular aneurysm repair: clinical evaluation of a new automated three-dimensional software. Ann Vasc Surg 2010;24:912-920.

23 Rueckert D, Frangi AF, Schnabel JA: Automatic construction of 3-D statistical deformation models of the brain using nonrigid registration. IEEE Trans Med Imaging 2003;22:1014-1025.

24 Xu C, Prince JL: Snakes, shapes, and gradient vector flow. IEEE Trans Image Process Publ IEEE Signal Process Soc 1998;7:359-369. 
25 Melnick G, Guerios EE, Agreli G: Modular aortic valve prosthesis for transcatheter aortic valve implantation: a novel concept with a new implantation method. Minim Invasive Ther Allied Technol MITAT Off J Soc Minim Invasive Ther 2017;26:60-64.

26 Elattar M, Wiegerinck E, van Kesteren F, Dubois L, Planken N, Vanbavel E, et al.: Automatic aortic root landmark detection in CTA images for preprocedural planning of transcatheter aortic valve implantation. Int J Cardiovasc Imaging 2016;32:501-511.

27 Liang L, Martin C, Wang Q, Sun W, Duncan J: Estimation of aortic valve leaflets from 3D CT images using local shape dictionaries and linear coding2016, pp 978432-9784329. 